\title{
THE FRAMEWORK OF THE EMOTION OF ARTIFICIAL INTELLIGENCE IN THE ROBOTIC SYSTEM - AN OPPORTUNITY FROM DISRUPTION
}

\author{
Ms Joyce Mary B, Ms Sparsha Hoskonde, Ms Kruthika, Ms Alaikya \\ Department of Computer Science Engineering, \\ Presidency University, India
}

\begin{abstract}
The drastic advancement and spin out technologies to respond artificially to the stimulus has made the man kind to wonder. The demand and logical approach has legitimated for the further integration of the robotic system in the human firmament. In order to advance the robotic system and to symbolize the problems possessed in the stipulated time, the researchers have made a great contribution in the field of development and further fragmentation of the algorithms. In order to achieve and make the robotic system to have a better communication with the humans, there is a requirement of the artificial intelligence to revolutionize in the recognition and understanding of human emotions in the conceptual way and also to anticipate and respond in the appropriate ways depending on the situation smoothly. This paper approaches on the development of the emotions of the robotic system which uses the technique of quantum computing mechanism in virtualization with the dynamic load balancing using Recurrent Neural Networks for the better manifesting response and flexibility. This paper also speaks about the recommendation and provides emphasis and glimpse on how the present world should enhance and develop in virtualization of artificial intelligence.
\end{abstract}

Keywords-- Artificial Intelligence, Quantum Computing Mechanism, Virtualization, Dynamic load balancing, Recurrent Neural Networks.

\section{INTRODUCTION}

As the world is heading towards the development of the artificial intelligence, there is a great importance given for the emotions so to merely make the human life much more efficient to communicate with the sustainable development in the robotic architectural system.

Since the evolution of the artificial intelligence, there is a great revolution which took place in the present contemporary world. Every move of a man is technologized in the way that the human life without artificial intelligence becomes unimaginable. To achieve in enhancing the sustainable development and to track the prosperity in the emotions of Robotic system towards the human, we would speak about the two phases which can go hand in hand. The one phase of the architecture should be off the dynamic balancing of Recurrent Neural Network and the other phase should be off Quantum Computer Mechanism using Virtualization. Therefore to uplift the present effective communication, we could encourage these both phases.

The artificial intelligence of the robotic system which was developed by David Hanson called Sofia is really the mysterious concept in the human world. As we all have known about how she has got citizenship in Saudi Arabia, mesmerizes us to just think of how she could even respond to the human stimuli. Obviously, as a common civilian we would justify ourselves thinking, as the way how the computer Google respond to you when you appeal any questions to find out the answers. And obviously the AI specialists would know that it would be typically from Recurrent Neural Network. This paper gives a complete approach of how the Recurrent Neural Network should balance with Quantum Computing in Virtualization in uplifting the effectiveness of emotions.

[Image-1]

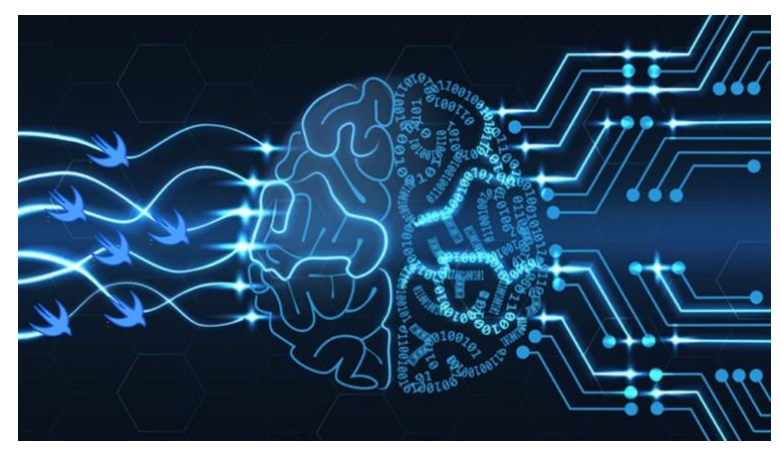

Fig .1 Digital Neuron Connections 


\section{International Journal of Engineering Applied Sciences and Technology, 2020 \\ Vol. 5, Issue 1, ISSN No. 2455-2143, Pages 328-340 \\ Published Online May 2020 in IJEAST (http://www.ijeast.com)}

\section{THE IMPORTANCE OF THE PHASES}

A. Development of Quantum Computing Mechanism with Storage Network:

- Quantum Computing basically works on the properties of subatomic particles which can perform certain kind of calculations exponentially.

- Provided with Bayes' rule of decision interface which will make the robotic system to distinguish the answers probably whether it is right or wrong after the recognition process.

- The embedded photons, electrons would play a major role in the fast identification of the solution for the complex problems

- Since the Quantum Computing directly deals with the architectural integrated circuits, the virtualization if provided would bring out the essence of autonomous robotic system to be advanced more.

- It also helps the robotic system to break down the encrypted system.

- Introducing the storage network in the system brings out the effectiveness.

- Any robotic system for that matter to improve its calculation when it is compared with clock cycle, Quantum Computing plays a very important role in the fastest complex calculation.

- The configuration of Virtual environment is created for bringing realistic for the robot to check and identify the problematic statement which arises within itself.

- Finding the matching phone number in a list of a billion entries would require just 31,623 operations - the square root of a billion - and, obviously, a small fraction of the time ${ }^{[2]}$ the robotic system would take, if acquired with Quantum mechanism with virtualization.

- Using Virtual file system there is merely back-up and recovery which would indeed help the robotic to recognize in the stipulated time.

- To recognize the sophisticated images, even the sensors require Quantum Mechanics to provide the complete understanding about the image formation process.

B. Dynamic balancing of Recurrent Neural Network

- Recurrent Neural Network which has great ability to recognize the sequential of model and then depict and sense with the hidden neuron makes the robotic system recognize and response to the humans.

- There are hidden layers also present in the Recurrent Neural Network (RNN).
- It also plays a very important role in the suboptimal as communication medium too to develop the emotion of robotics through these techniques.

- It can support by interpreting the language so that it makes the robotic system to have effective communication with the humans.

- The use of Pre-emphasis and windowing in future development with the software in the robotic system is very effective.

- The Pre-emphasis which helps in the spectrum voice segment in high frequencies.

- The dynamic balancing of this Recurrent Neural Network with the Quantum Computing Mechanism can uplift the emotion of the robotic system efficiently.

\section{Benefits of the Recurrent Neural Network}

- Recurrent Neutral Network allows operating over sequences of vectors.

- They are more efficient than Vanilla Neural Network

- Automatic transcription takes place

- Automatic recognition in the robotic system

- It provides an environment for the human computer interface.

- An example of piece of discrete code where input and output vectors are showcased.

$\mathrm{rnn}=\mathrm{RNN}($ )

$\mathrm{y}=\operatorname{rnn} . \operatorname{step}(\mathrm{x}) \# \mathrm{x}$ is an input vector, $\mathrm{y}$ is the RNN's output vector ${ }^{[3]}$.

D. Benefits of the Quantum Computing Mechanism

- There are lots of pros because of the behavior of molecules.

- It also includes the semiconductor behavior

- It is so faster that it can solve the problems in few milliseconds.

- Cloud Computing too plays a major role.

- It also breaks the encrypted system

- Eliminates Energy.

\section{DISCUSSION}

As the world is mesmerized by the robotic system Sofia who got citizenship in Saudi Arabia is able to showcase the facial expression, artificial emotions, personality and social emotions due to pre-scripted code which in turn the electrical signals plays a very important role. The different sensors which are used for identification and typically recognitions are Vision Image Sensor and Tactile Sensor. So therefore 


\section{International Journal of Engineering Applied Sciences and Technology, 2020 \\ Vol. 5, Issue 1, ISSN No. 2455-2143, Pages 328-340 \\ Published Online May 2020 in IJEAST (http://www.ijeast.com)}

when we use the architectural of the fig 1, obviously the preexisting program with the electrical signals would make a drastic difference with the quantum computing mechanism and the Recurrent Neural Network to uplift the advanced emotions. If this kind of architecture is adopted, then would be drastic improvement.

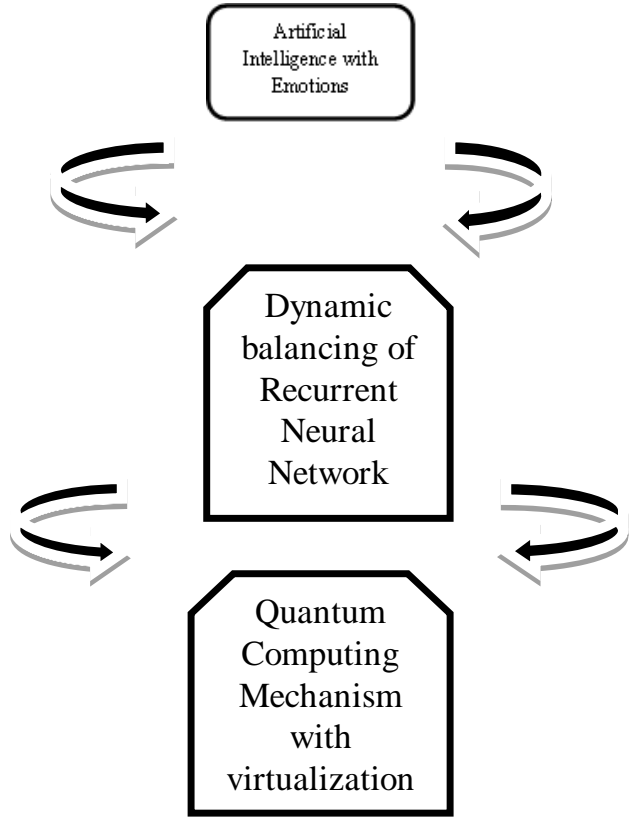

Fig. 2. Cluster in development of emotion

\section{EQUATION}

Here is a sample for demonstrating the modelling with an equation.

Recurrent Neural Network Language Modelling

Step 1: Previous word using 1 -of $-\mathrm{N}$ for encoding for inputting layer

$(000 \ldots 001000 \ldots) \longrightarrow$ Vocabulary size

Step 2: Recursive structure for hidden layer using logic unit

$$
\mathrm{f}(\mathrm{z})=\frac{1}{1+e^{-z}}
$$

Step 3: Probability distribution of the next word for output layer

$$
\mathrm{g}\left(Z_{k}\right)=\frac{e^{z_{k}}}{\sum_{k} e^{z_{k}}}
$$

\section{CONCLUSION}

The digital world is enhancing very drastically showcasing its decision 0 or 1 (True/False). Therefore to make the human life more advanced and bring the automation in this current era will really pacify him. Therefore this architecture surely will make the robotic system to respond as quickly as possible not taking more than number of milliseconds for the successive communication. At the same time having eager for security is very essential. The robots are indeed slaves to the man kind; it should not occupy the human's domains.

\section{REFERENCES}

[1][Image 1]

Y.-B. Lin, Y.-J. Shih, H.-C. Tseng, and L.-J. Chen, "LWA rate adaption by enhanced event-triggered reporting," IEEE Trans. Veh. Technol., vol. 67, no. 11, pp. 10950_10959, Nov. 2018.

[2] Y.-W. Lin, Y.-B. Lin, M.-T. Yang, and J.-H. Lin, "ArduTalk: An arduino network application development platform based on IoTtalk," IEEE Syst. J., vol. 13, no. 1, pp. 468_476, Mar. 2019.

[3] K. R. S. R. Raju and G. H. K. Varma, "Knowledge based real time monitoring system for aquaculture using IoT," in Proc. IEEE 7th Int. Adv. Comput. Conf., Jan. 2017, pp. 318_321.

[4] Y.-B. Lin, L.-K. Chen, M.-Z. Shieh, Y.-W. Lin, and T.-H. Yen, "CampusTalk: IoT devices and their interesting features on campus applications," IEEE Access, vol. 6, pp. 26036_26046, 2018.

[5] T. Masaharu, et. al., "Effect of temperature on survival, growth and malformation of cultured larvae and juveniles of the seven-band grouper epinephelus septemfasciatus," Fisheries Sci., vol. 80, no. 1, pp.69_81, 2014

[6] Y.-B. Lin, Y.-W. Lin, C.-M. Huang, C.-Y. Chih, and P. Lin, "IoTtalk: A management platform for recon_gurable sensor devices," IEEE Internet Things J., vol. 4, no. 5, pp. 1152_1562, Oct. 2017.

[7] Y.-B. Lin, et. al., "Easyconnect: A management system for IoT devices and its applications for interactive design and art," IEEE Internet Things J., vol. 2, no. 6, pp. 551_561, Dec. 2015.

[8] Y.-W. Lin, Y.-B. Lin, C.-Y. Hsiao, and Y.-Y. Wang, "IoTtalk-RC: Sensors as universal remote control for aftermarket home appliances," IEEE Internet Things J., vol. 4, no. 4, pp. 1104_1112, Aug. 2017.

[9] K. M. Stehfest, C. G. Carter, J. D. McAllister, J. D. Ross, and J. M. Semmens, "Response of atlantic salmon salmo salar to temperature and dissolved oxygen extremes established using animal-borne environmental sensors," Nature, vol. 7, no. 1, p. 4545, 2017.

[10] C. Encinas, E. Ruiz, J. Cortez, and A. Espinoza, "Design and implementtation of a distributed IoT system for the monitoring of water quality in aquaculture," in Proc. Wireless Telecommun. Symp. (WTS), Apr. 2017, pp. 1_7. 